\title{
Proposta de Aplicativo em Dispositivos Móveis para Ensino de Leitura Baseado no Controle por Unidades Mínimas
}

\author{
Roberto Antonio da Silva ${ }^{1}$, Edson Pinheiro Pimentel ${ }^{2}$ \\ ${ }^{1}$ Mestrando em Engenharia da Informação - Universidade Federal do ABC (UFABC) \\ Santo André - SP - Brasil \\ ${ }^{2}$ Professor da Universidade Federal do ABC (UFABC) - Centro de Matemática, \\ Computação e Cognição (CMCC) \\ 09090-400 Santo André - SP - Brasil \\ roberto.antoniodufabc.edu.br, edson.pimentel@ufabc.edu.br
}

\begin{abstract}
In behavior analysis, two paradigms are useful for development programs of teaching reading and writing: the stimulus equivalence, which search for identify relationships between stimuli in the teaching of reading, and the smaller units than the word, from the Skinner concept of control by minimal units. In this context, computer systems can help in use these paradigms through the collection, storage and presentation of data in an automated way. This article meant to present the proposed of the application m-EQUIV for mobile devices, which has the purpose of support the teaching of reading based on control by minimal units. The main purpose of m-EQUIV environment is take to the classroom a gamification environment that can be used from mobile devices to support the teacher in the task of teach children to read.
\end{abstract}

Resumo. Na análise do comportamento, dois paradigmas têm sido úteis para a elaboração de programas de ensino de leitura e escrita: o de equivalência de estímulos que busca identificar relações entre estímulos no ensino da leitura e o de unidades menores do que a palavra, a partir do conceito de Skinner de controle por unidades mínimas. Neste contexto, sistemas computacionais podem ajudar no uso desses paradigmas realizando a coleta, armazenamento e apresentação dos dados de forma automatizada. Este artigo tem por objetivo apresentar a proposta do aplicativo m-EQUIV, para dispositivos móveis que tem por finalidade apoiar o ensino de leitura baseado no controle por unidades mínimas. O principal propósito do ambiente m-EQUIV é levar para a sala de aula um ambiente gamificado, que possa ser utilizado a partir de dispositivos móveis, de modo a apoiar o professor no ensino de leitura para crianças.

\section{Introdução}

A leitura é um dos elementos cruciais para a prática consciente da sociabilidade e do desenvolvimento de um indivíduo. Possui fundamental importância para a vida das pessoas, estando presente na maior parte das atividades cotidianas. Através da leitura é possível o exercício da cidadania, permitindo que o leitor tenha o raciocínio lógico, à reflexão crítica e a mudança de atitude, pois a leitura é uma porta aberta para uma melhor compreensão do mundo. Ler é saber interpretar e reconhecer os sentidos das palavras é saber diferenciar o contexto do que foi lido. Para Kleiman (2011) a leitura é um processo que se evidencia por meio da interação entre os diversos níveis de conhecimento do leitor: 
o conhecimento linguístico, o conhecimento textual e o conhecimento de mundo, ou seja, a leitura é adquirida com o tempo, de forma sistematizada e com a prática.

$\mathrm{Na}$ análise do comportamento, dois paradigmas têm sido úteis para a elaboração de programas de ensino de leitura e escrita: o de equivalência de estímulos que objetiva identificar relações entre estímulos no ensino da leitura e o de unidades menores do que a palavra, a partir do conceito de Skinner de "controle por unidades mínimas" [Skinner 1957]. Através do modelo da equivalência de estímulos proposto por Sidman e Tailby (1982) foi possível estabelecer critérios operacionais permitindo a identificação de relações simbólicas e simular a aquisição destas em laboratório, como por exemplo: uma criança escolher um entre dois ou mais estímulos de comparação condicionalmente à apresentação de um estímulo modelo. Este trabalho baseia-se na elaboração de programas de ensino de leitura e escrita utilizando a equivalência de estímulos que busca identificar relações entre estímulos no ensino da leitura por controle por unidades mínimas.

Parte-se da perspectiva de que é possível utilizar as tecnologias digitais para apoiar o ensino de leitura para crianças. A fim de possibilitar mais mobilidade e flexibilidade no contexto educativo da sala de aula, vislumbra-se o uso de dispositivos móveis, pois a aprendizagem, por meio destes equipamentos, é um campo fértil para investigação, no meio educacional que explora essencialmente a mobilidade e a interatividade, a facilidade de uso é outro aspecto de grande influência [Werbach e Hunter 2012].

Com a redução dos custos e a popularização dos dispositivos móveis, assim como a disseminação da gamificação é possível apresentar novas perspectivas para a informática educativa, pois para a criança o aprendizado na forma de jogos desenvolve suas habilidades e conhecimentos, tornado o aprendizado mais prazeroso. Diante deste cenário está em fase de desenvolvimento o m-EQUIV, um software que pretende ser uma plataforma computacional gamificada para dispositivos móveis objetivando auxiliar o ensino da leitura em crianças através da pesquisa em equivalência e controle por unidades mínimas. Este artigo está organizado conforme segue: a seção 2 apresenta a fundamentação teórica para a elaboração este trabalho. Na seção 3 está descrito os trabalhos correlatos. Na seção 4 está descrito a proposta preliminar do desenvolvimento do software m-EQUIV. E na seção 5 as considerações finais.

\section{Fundamentação Teórica}

Os estudos iniciais apoiaram-se no levantamento de literatura para identificar ferramentas com propósitos semelhantes ao software que está em desenvolvimento. O objetivo desta seção é atribuir referenciais teóricos para a compreensão da proposta apresentada neste trabalho, onde inicialmente é apresentado sobre leitura, e em seguida dispositivos móveis e por último gamificação.

\subsection{Ensino de Leitura e o Controle por Unidades Mínimas}

A leitura é a capacidade que vai da decodificação do sistema de escrita até a compreensão e a produção do sentido para o texto lido, como objetivo principal a compreendê-lo. De acordo com Foucambert (1994), o aprendizado da leitura é um dos desafios da escola e, talvez, o mais valorizado e exigido pela sociedade. Cagliari (2004) afirma que a leitura é a atividade mais importante e que serve de base para as outras atividades escolares, pelo fato da ligação da mesma com tudo que é ensinado na escola. Assim, deve-se ter em mente que formar leitores não é somente ensinar a decodificar os signos, é dar condições 
de ir mais além, ajudando no desenvolvimento de estratégias que os possibilitem alcançarem uma aprendizagem significativa.

Pois para que um indivíduo torne-se capaz de ler praticamente qualquer palavra, é necessário apenas a aprendizagem de um pequeno conjunto de unidades mínimas (letras e silabas), e a aprendizagem do desempenho de combinação e recombinação destas unidades mínimas em unidades maiores (palavras e sentenças). O sistema alfabético possibilita o desenvolvimento de um repertório de unidades mínimas [Skinner 1957] que podem ser recombinadas na leitura de novas palavras. A formação e o encadeamento deste repertório de unidades mínimas requer que o estudante aprenda a responder sob controle dos caracteres específicos e de sua disposição relativa. O procedimento proposto por Sidman [1971] pode desenvolver o controle de unidades mínimas por meio de treino e ensino de algumas palavras, proporcionando dessa forma, a aquisição da leitura com compreensão pela criança, sob controle de alguns de seus componentes.

Unidades mínimas textuais (letras e sílabas) são importantes fatores de controle no repertório do aprendiz iniciante [De Rose et al. 1989] [Hübner et al. 1993]. Essas relações não são ensinadas direta ou explicitamente, mas emergem a partir do ensino de relações condicionais que lhes dão suporte. Os estímulos são formados por A (som) B (imagem) e C (escrita), assim é possível estabelecer a reflexividade se o participante relacionar cada estímulo a ele mesmo, sem treino explícito: A com A, B com B, C com C. A simetria é demonstrada quando o participante, após aprender a selecionar o estímulo de escolha $\mathrm{C}$, diante do estímulo-modelo B (relação BC), faz também o inverso, sem treino específico para tal: diante do estímulo-modelo $\mathrm{C}$, escolhe o estímulo $\mathrm{B}$ (relação CB). Já a transitividade é demonstrada se o participante, após aprender as duas relações condicionais AB e AC, é capaz de demonstrar a relação BC ou CB [Matos et al. 2002].

De acordo com Matos et al. [2002], através do pareamento entre estes estímulos uma criança consegue ler com compreensão, pois consegue fazer relações entre a palavra escrita, o desenho de um objeto (ou até mesmo o próprio objeto) e a palavra ouvida. A figura 2.1 mostra as relações de equivalência, onde as linhas contínuas são as relações ensinadas, e as linhas tracejadas são as relações emergentes testadas.

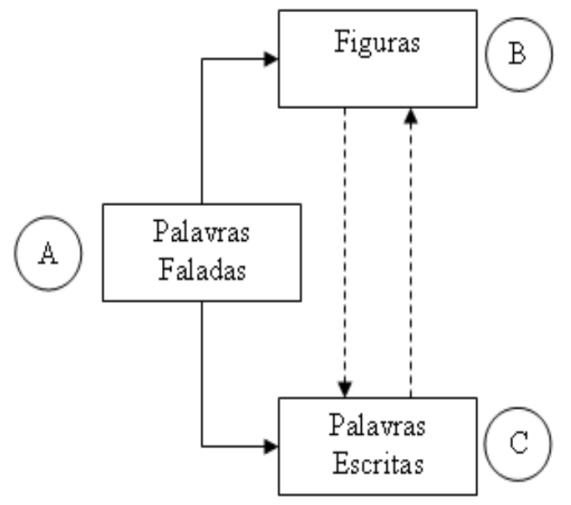

Figura 2.1. Estudo de leitura recombinativa. Fonte: Matos et al [2002]

Aprimorando esse estudo, Matos et al. (2002) decidiram por fragmentar as palavras dos treinos em elementos silábicos, e em seguida, recombinar estes elementos gerando novas palavras, e associando a estas as novas figuras correspondentes, conforme figura 2.2 . 


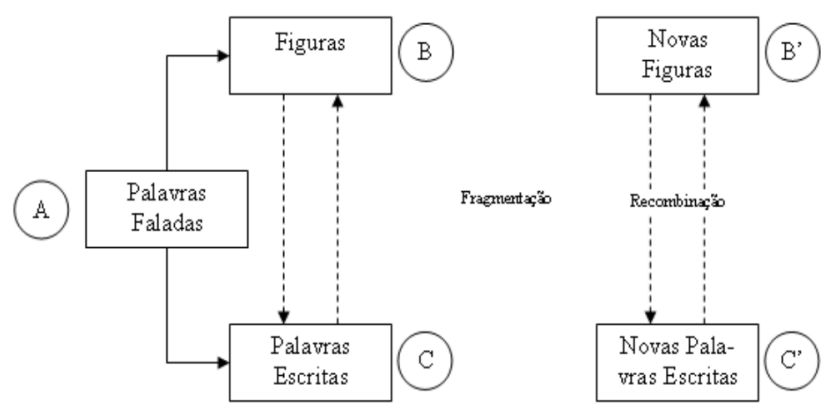

\section{Figura 2.2. Estudo de leitura recombinativa após a fragmentação e recombinação. Fonte: Matos et al [2002]}

O modelo de equivalência de estímulos trouxe notáveis avanços para o tratamento empírico da formação e manipulação de símbolos, fornecendo critérios que permitem uma definição empírica de "compreensão". Desta maneira o processo de ensino/aprendizagem é baseado nas mesmas intervenções: avaliação, observação, análise e o uso dessas informações para delinear programas fundamentados na motivação e individualização.

\subsection{Dispositivos Móveis}

Dispositivos móveis são aparelhos que têm se tornado cada vez mais populares e capazes de executar as ações realizadas em computadores pessoais, como navegar na internet, acessar emails e redes sociais, e executar diferentes tipos de programas. De acordo com Firtman (2010) dispositivos móveis são aqueles que possuem as seguintes características: i) portátil: que possa ser carregado sem muito esforço, como um molho de chaves ou uma carteira; ii) pessoal: cada pessoa tem seu próprio dispositivo, com suas customizações e normalmente não se compartilha sua utilização; iii) companheiro: é possível estar com o dispositivo o tempo todo; iv) fácil de usar: no sentido de condições de uso, como por exemplo, não é necessário utilizar o dispositivo sentado, ou num lugar com ótima iluminação, ou ter que esperar dois minutos para que o sistema esteja pronto para uso; v) conectado: deve ser possível conectar na internet quando necessário.

Desta maneira a possibilidade de utilizar dispositivos móveis no processo de ensino e aprendizagem permite uma flexibilidade para o aluno executar as atividades educacionais em qualquer lugar e a qualquer hora. Permitindo assim que os softwares antes desenvolvidos para computadores de mesa estejam disponíveis para este tipo de equipamento, facilitando o acesso a conteúdos educacionais. Neste contexto podemos citar o smartphones que são aparelhos bastante difundidos no mercado e com preços relativamente acessíveis, permitem que diferentes pessoas, e de diferentes classes sociais, tenham acesso aos conteúdos educacionais.

\subsection{Gamificação}

Atualmente estamos vivenciando o surgimento de um novo acontecimento denominado gamificação [Werbach e Hunter 2012], que se baseia na utilização dos componentes dos jogos de videogames fora do ambiente de jogos, com o intuito de motivar as pessoas à ação de auxiliar na solução de problemas e estimular a aprendizagem [Kapp 2012]. Assim o objetivo da gamificação é criar um ciclo de expectativa de recompensa comum em jogos 
para manter o usuário engajado com o produto.

$\mathrm{Na}$ área da educação a gamificação possui grande potencial para ser aplicado, pois de acordo com Lee (2011), é possível não apenas ensinar com jogos ou através deles, mas sim usar elementos destes como forma de promover a motivação e o envolvimento dos alunos. Assim escolas estão incorporando jogos educacionais na sala de aula com o objetivo de atrair mais a atenção dos alunos. Neste sentido Fardo (2013) diz que a sala de aula pode ser equiparada a um jogo, pois a gamificação é um fenômeno que consiste no uso de elementos, estratégias e pensamentos dos games fora do contexto de um game, com a finalidade de contribuir para a resolução de algum problema. Assim a gamificação é uma tendência e pode ter uma grande eficiência na educação, pelo rápido feedback e boa atratividade para as crianças que estão imersas cada vez mais nas tecnologias digitais.

\section{Trabalhos Correlatos}

Atualmente existem diversos softwares educacionais para auxiliar o ensino de crianças, alguns baseados na pesquisa em equivalência como o EQUIVIUM [Pimentel 2009] e outros direcionados para dispositivos móveis, dentre eles, o Universo Móvel [Neves 2014], que é um aplicativo para aprendizagem de astronomia. Porém não se identificou nenhum que utilize a gamificação em dispositivos móveis para aprendizagem da leitura.

\subsection{Software Equivium}

O EQUIVIUM [Pimentel 2009] é um software que foi desenvolvido para o Grupo de Pesquisa Instituto Nacional de Ciência e Tecnologia (INCT) - Estudos sobre o comportamento, cognição e ensino (ECCE) coordenado pela Professora Dra. Martha Hubner sob orientação do Prof. Dr. Edson Pimentel, com o objetivo de realizar a coleta de dados e dar suporte as etapas relacionadas ao ensino de leitura com compreensão baseado na pesquisa em equivalência e Controle por Unidades Mínimas. Este software possui sua utilização consolidada, devido a sua utilização por pesquisadores [Coelho 2013], atendendo assim seu propósito, de auxiliar o ensino da leitura através da automatização da coleta dos dados e aumento da produtividade nas etapas envolvidas no ensino de leitura com compreensão baseado na pesquisa em equivalência e controle por unidades mínimas.

Assim com a utilização do EQUIVIUM [Pimentel 2009] é possível trazer benefícios para a pesquisa em equivalência e controle por unidades mínimas, permitindo a organização dos dados. Pois este software oferece flexibilidade para a realização das configurações, cadastramento de novos estímulos, coleta dos dados de forma segura e sem erros, e o armazenamento e a apresentação dos dados na forma de relatório. Contribuindo para a identificação dos pontos em que a criança tem dificuldades.

\subsection{Universo Móvel}

O Universo Móvel [Neves 2014] é um aplicativo educacional desenvolvido para dispositivos móveis para apoiar o ensino da astronomia no ensino fundamental e médio. Este aplicativo foi desenvolvido utilizando diferentes mídias digitais como sons e imagens, e seu conteúdo bibliográfico foi criado a partir de estudos e publicações de instituições de ensino e pesquisa, como o Departamento de Astronomia do Instituto de Física da Universidade Federal do Rio Grande Sul (UFRGS).

Este aplicativo possui entre outras, as ferramentas denominadas: “Astro Quiz", 
módulo que permite ao aluno avaliar seu conhecimento prévio e/ou aprendido; e a ferramenta "Instrumentos Astronômicos" que foca no aprendizado dos equipamentos utilizados pela astronomia. Assim com a utilização destas ferramentas é possível uma maior interatividade entre os alunos e o aplicativo, tornando o ensino de noções da astronomia mais dinâmico e lúdico. Para o desenvolvimento deste software foram utilizados as linguagens HTML5 e JavaScript, além CSS3, e para a melhor compatibilidade com diversos tipos e modelos de dispositivos móveis, foram utilizados os framework JQuery/JQuery Mobile. Este aplicativo esta disponibilizado em formato aberto e com licença permissiva, o que ajuda a disseminar a filosofia do software livre nas tecnologias móveis. Assim o Universo Móvel [Neves 2014] é um aplicativo para dispositivos móveis objetivando aproximar jovens e adolescentes as noções da astronomia através de tecnologias móveis.

\section{4. m-EQUIV}

O ambiente m-EQUIV está em desenvolvimento, com o objetivo de ser um aplicativo para dispositivos móveis (smartphones e tablets), visando auxiliar o ensino da leitura utilizando como base a educação, tecnologia, e a gamificação. Este ambiente contém componentes como design e usabilidade, com o objetivo de estimular a imaginação da criança, através da utilização de recursos da gamificação motivando o aluno a concluir todas as etapas da atividade. Na figura 4.1 é apresentada a arquitetura do m-EQUIV.

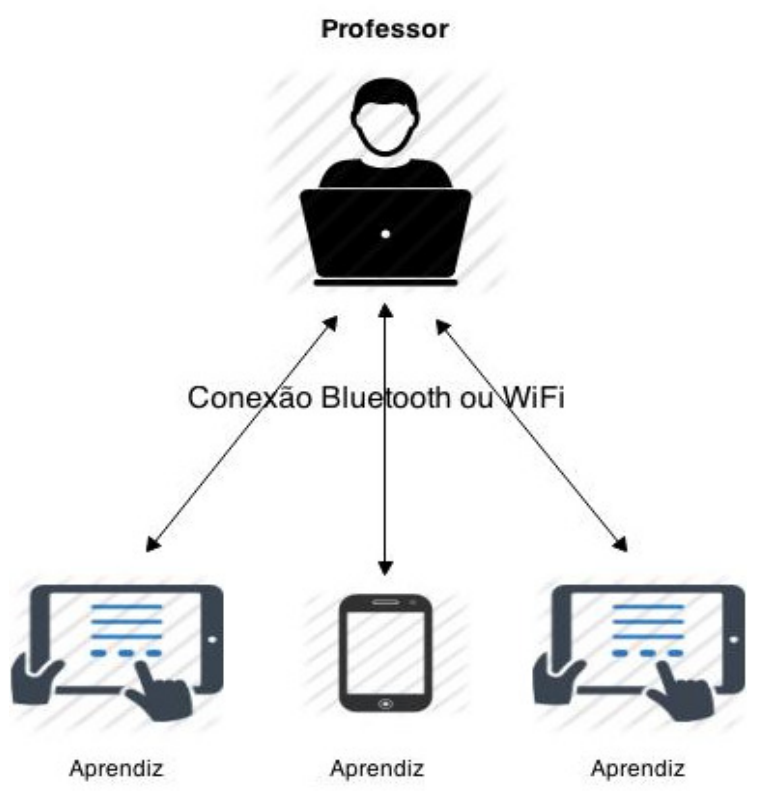

Figura 4.1 - Arquitetura do Software

O m-EQUIV pretende torna-se uma ferramenta de apoio que utiliza textos, sons, imagens e animações para motivar o aluno na aprendizagem da leitura e estimular a criança para realizar mais atividades em dispositivos móveis.

\subsection{Levantamento de requisitos}

Esta atividade tem como objetivo compreender o problema, dando a visão do que deve ser construído. O levantamento de requisitos para a criação do m-EQUIV foi realizado através do estudo do software EQUIVIUM [Pimentel 2009] e de seus requisitos, com o intuito de entender seu funcionamento e sua modelagem. 


\subsubsection{Requisitos}

Os principais requisitos funcionais do m-EQUIV são listados a seguir:

- Professor: compreende os dados dos professores cadastrados para utilizar o ambiente;

- Aprendiz: são os dados referentes ao aprendiz;

- Reforço sonoro: recurso sonoro para motivar o aprendiz;

- Reforço visual: recurso visual apresentado ao final para motivar o aprendiz;

- Estímulo: objetos manipulados pelo software para aplicação da atividade;

- Tentativa: são as combinações de estímulos-modelo e estímulos-escolha;

- Fase: é o conjunto de tentativas, e através das fases que o professor monta a sequência da atividade;

- Sessão: é o conjunto de fases que a serem aplicadas aos aprendizes;

- Histórico: é o conjunto de dados referentes às sessões realizadas;

- Anagrama: é conjunto de combinações de estímulo-modelo, estímulo-escolha e estímulos extras para a realização de um Anagrama Silábico.

Os principais requisitos não-funcionais do m-EQUIV, ou seja, as propriedades do sistema, suas restrições e qualidades globais são listados a seguir:

- O m-EQUIV deverá ser acessado a partir de dispositivos moveis como smartphones e tablets, tanto pelo professor como pelos aprendizes;

- O m-EQUIV também poderá ser acessado em desktops e notebooks, principalmente para facilitar a realização de cadastros e configurações pelo professor;

- A sincronização dos dados será realizada entre os dispositivos do professor e dos alunos de modo batch, ou seja, apenas quando os mesmos estiverem conectados através de conexão sem fio.

\subsubsection{Modelo lógico}

Através da diagramação de todos os respectivos componentes, é possível ter a real ideia do papel exercido por cada integrante, tanto para a elaboração das atividades, quanto na execução das mesmas. Assim a figura 4.2 demonstra o diagrama de caso de uso indicando a participação de cada ator envolvido na utilização do software.

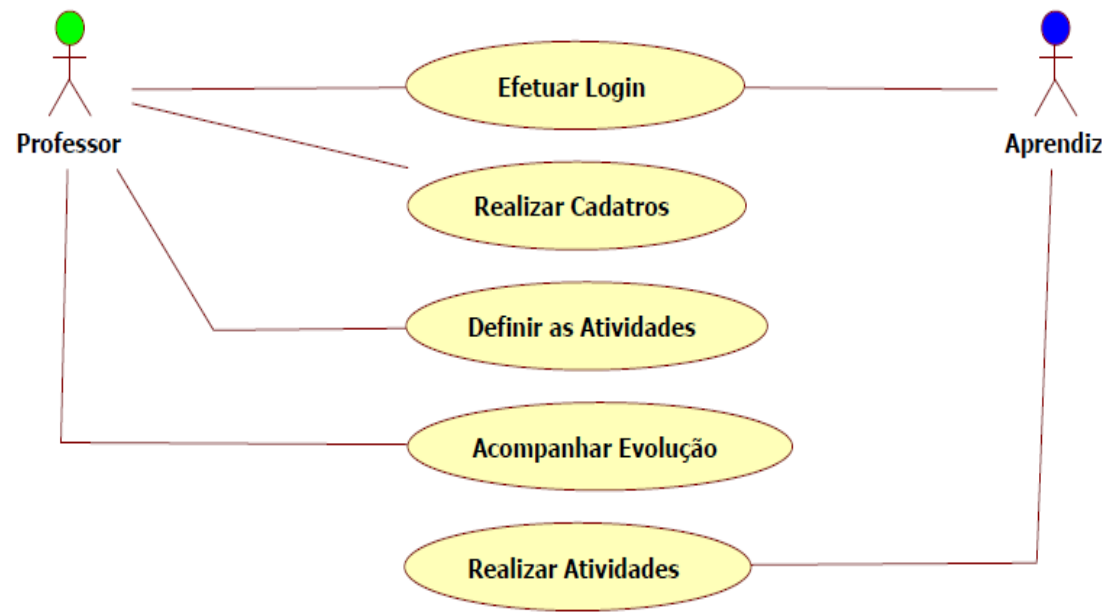

Figura 4.2 - Diagrama de caso 


\subsection{Modelo preliminar}

No modelo preliminar do m-EQUIV o professor é responsável por realizar todos os cadastros, criar as atividades, realizar a avaliação da criança, e enviar e receber os dados para os dispositivos móveis. Ficando para a criança a responsabilidade de realização das atividades. Com o design de telas amigáveis e sensíveis ao toque, conforme a figura 4.3, é possível tocar e arrastar as figuras, letras ou sílabas para a realização das atividades, pois a utilização da tela sensível ao toque, e a ação de acessa-las ou move-las utilizando apenas o dedo, torna a tarefa de aprendizado simples e rapidamente assimilada pela criança.

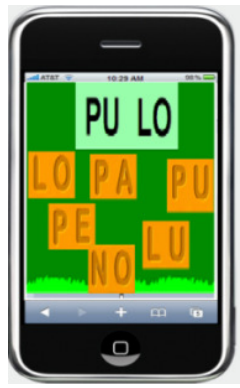

Figura 4.3 - Demonstração da execução de uma sessão

A sequência para a realização das atividades será baseada na gamificação, ou seja, após definido as fases pelo professor o software irá montar um jogo com as atividades definidas, assim ao final de cada atividade será apresentado uma tela, figura 4.4, mostrando ao aluno a conclusão da fase e também seu avanço com uma gratificação.

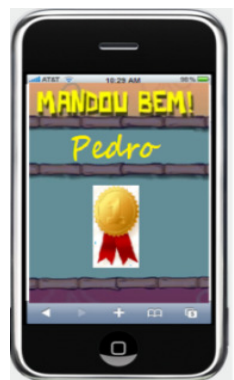

Figura 4.4 - Demonstração tela de finalização de fases

Pretende-se com este novo recurso incentivar a criança a continuar realizando seu aprendizado de forma mais prazerosa, pois conforme o tipo de atividade que será realizada poderá executar diversas fases, conforme o exemplo da pesquisa de Coelho (2013) que utilizou 12 fases. O m-EQUIV dispõe ainda de novas funcionalidades, sugeridas por pesquisadores que utilizaram o EQUIVIUM [Pimentel 2009] em suas pesquisas [Coelho 2013], melhorando desta forma a interação da criança com os dispositivos computacionais. Para isso o m-EQUIV é divido em dois módulos distintos devido a sua complexidade: Módulo Professor e Módulo Aprendiz, conforme segue.

\subsection{Módulo professor}

No módulo professor será possível realizar todos os cadastros necessários para a utilização do software, assim como verificar a evolução da criança.

\subsubsection{Cadastro}

Neste módulo serão realizados os oito cadastros básicos: professores, crianças, reforços, 
estímulos, tentativa, fase, sessão e anagrama, onde os seis primeiros cadastros são imprescindíveis, pois sem eles não será possível realizar uma sessão. Outro cadastro importante é o cadastro de estímulos, sendo este um dos itens principais para o m-EQUIV, pois nele estão contidos os elementos que o software manipula para a realização das atividades. Estes estímulos são de três tipos: áudio, imagem, e formação de componente para fragmentação este estímulo.

\subsubsection{Evolução}

Nesta parte será possível para o professor avaliar a evolução da criança. Através deste módulo o professor consegue identificar o avanço do aluno através do gráfico de pontos obtidos, e através dos relatórios, onde ambas as formas de avaliação serão baseadas dos dados coletados durante as atividades realizadas.

\subsection{Módulo aprendiz}

O módulo aprendiz é parte do software onde se realiza as atividades: sessão e anagrama cadastrados pelo professor. Este módulo é o motor principal do software, pois é neste módulo que as atividades serão realizadas, ou seja, é neste módulo que as atividades serão apresentadas ao aluno e as respostas serão armazenadas, para posterior análise do processo de aprendizagem. Nesta etapa o software substituirá as tarefas que o professor realizava de forma manual. Como a escolha da fase, aleatorização de estímulos, apresentação e ordenação visual destes estímulos, anotação de qual foi o conjunto de estímulo-modelo e estímulos-escolha formado em cada iteração de uma instância, além de totalizações e porcentagem de acertos. Desta maneira a coleta dos dados será realiza em "segundo plano", ou seja, enquanto a atividade é realizada os dados são armazenados sem nenhuma interferência.

\subsection{Tecnologias}

As tecnologias utilizadas para o desenvolvimento do m-EQUIV são softwares de código aberto (open source, em Inglês), ou seja, são softwares de utilização livre, cuja licença não é cobrada. As linguagens utilizadas são: HTML5 (Hyper Text Markup Languagem), CSS3 (Cascading Style Sheets) e JavaScript. Pois o uso destas tecnologias permite a execução do m-EQUIV em diferentes sistemas operacionais, incluindo Android e Windows, contribuindo para que o ambiente torne-se uma ferramenta de aprendizagem, disponível para todos.

\section{Considerações finais}

O ambiente m-EQUIV, descrito neste artigo encontra-se em fase de desenvolvimento inicial. Pretende-se através da utilização deste ambiente criar uma ferramenta que auxilie os professores a criarem oportunidades educacionais para todas as crianças. Pois através da flexibilidade que o m-EQUIV irá proporcionar ajudará professores a construírem tarefas de acordo com as necessidades individuais de cada criança, além de otimizar o tempo gasto para elaboração e análise das mesmas.

Assim o m-EQUIV pretende agregar valores como ferramenta didática em dispositivos móveis, contribuindo para uma aprendizagem mais significativa da leitura em sala de aula, utilizando com base a gameficação, com o objetivo de aumentar o interesse da criança, a aprender enquanto se diverte. 


\section{Referencias}

Coelho, A. S. (2013) Efeito do Treino e Teste de Palavras Monossilábicas no Responder Diferencial às Unidades Mínimas em Leitura de palavras Dissílabas. Tese Doutorado - Programa de Pós-Graduação em Psicologia. Instituto de Psicologia da USP.

De Rose, J. C., Souza, D. G., Rossito, A. L. e De Rose, T. M. S. (1989) Aquisição de leitura após história de fracasso escolar: equivalência de estímulos e generalização. Psicologia: Teoria e Pesquisa, 5, 325-346.

Fardo, M. (2013) A gamificação como método: estudo de elementos dos games aplicados em processos de ensino e aprendizagem. Dissertação (Mestrado) - Universidade Caxias do Sul, Programa de Pós-Graduação em Educação.

Firtman, M. (2010) Programming the Mobile Web. Sebastopol: O'reilly Media.

Foucambert, J. (1994) A leitura em questão. Porto Alegre: Artes Médicas.

Fitzek, F., Reichart, F. (2007) Mobile phone programming: and its Application to Wireless Network. Dordrecht: Springer.

Hübner-D’Oliveira, M. M., Matos, M. A. (1993) Controle discriminativo na aquisição da leitura: efeito da repetição e variação na posição das sílabas e letras. Temas em Psicologia, 2, 99-108.

Kapp, K. (2012) The Gamification of Learning and Instruction: Game-based. Methods and Strategies for Training and Education. Pfeiffer

Kleiman, A. (2011) Texto e Leitor: aspectos cognitivos da leitura. 14. ed. São Paulo. Pontes.

Lee, J., Hammer, J. (2011) Gamification in Education: What, How, Why Bother? Academic Exchange Quarterly, 15(2).

Matos, M. A, Hübner-D’oliveira, M. M.; Serra, V. R. B. P.; Basaglia, A. E.; Avanzi, A. L. (2002) Redes de relações condicionais e leitura recombinativa: pesquisando o ensinar a ler. Arquivos Brasileiros de Psicologia n ${ }^{\circ}$ 54, pp. 284-303.

Neves, B. G. B.; Melo R. S.; Machado, A. F. (2014). Universo Móvel: um Aplicativo Educacional Livre para Dispositivos Móveis. WSL 2014 - Workshop de Software Livre. Porto Alegre. Brasil

Pimentel, E. D, Baldani, D., Piccolo, G., Hubner, M. (2009). Um Ambiente para o Ensino de Leitura baseado na Pesquisa em Equivalência e Controle por Unidades Mínimas XX Simpósio Brasileiro de Informática na Educação.

Sidman, M. (1971) Reading and auditory-visual equivalences. Journal of Speech and Hearing Research, v. 14, p. 5-13.

Sidman, M., \& Tailby, W. (1982) Conditional discrimination vs. matching to sample: An expansion of the testing paradigm. Journal of the Experimental Analysis of Behavior, $37,5-22$.

Skinner, B. F. (1957). Verbal behavior. New York: Appleton, Century, Crofs.

Werbach, K.; Hunter, D. (2012) For The Win! How game thinking can revolutionize your business. Filladélfia. Wharton Digital Press. 